\title{
Quantum seeded Sub-20 fs pulse train generation using transient SRS in H2-filled inhibited coupling HC-PCF
}

\author{
J. Ignacchiti ${ }^{1}$, D. Kergoustin ${ }^{1}$, F. Amrani ${ }^{1,2}$, B. Debord ${ }^{1,2}$, F. Gérôme ${ }^{1,2}$ and F. Benabid $^{1,2}$ \\ ${ }^{1}$ GPPMM Group, Xlim Institue, CNRS UMR 7252, Université de Limoges, 87060 Limoges, France. ${ }^{2}$ GLOphotonics, 123 Avenue \\ Albert Thomas, Limoges, France.f.benabid@xlim.fr
}

\begin{abstract}
We report on the generation of pulse trains from a hydrogen-filled hollow-core fiber pumped by 10 ps pulses for wave synthesis. Experimental results show ultrashort pulse trains separated by 57 fs with $20 \mathrm{fs}$ duration. (c) 2020 The Author(s)
\end{abstract}

\section{Introduction}

Ultra-short pulse generation is based on a broadband spectrum with phase locked spectral components. Among the physical processes that can engender ultra-broad coherent spectrum is Raman scattering provided the medium excitation is undertaken using specific schemes. For example, Harris et al [1] showed that a Raman medium can be transformed into a phase modulator with a modulation frequency set by the medium Raman resonance if the medium is excited with two pulsed lasers whose frequency difference is slightly detuned from the Raman resonance. This molecular modulation scheme led to the first demonstration of optical waveform synthesis over several octaves [2]. Transient Raman scattering can be an efficient means to generate phase locked spectral components and simpler alternative to the molecular modulation scheme if only one of the spatio-temporal modes (STM) of the spontaneously emitted Stokes field is amplified to the stimulated regime [3]. Indeed, the quantum noise initiated Stokes fields $E_{\mathrm{s}}$ can be expressed as a linear combination of spatial and temporal modes (i.e. $E_{s} \propto$ $\left.\sum_{n}\left[\psi_{n}(t) \sum_{k} \phi_{k}(r)\right]\right)$ [3], and if by a judicious excitation scheme one could select one and only one STM, it means that coherent Raman comb could be generated with a single laser excitation. This was experimentally demonstrated using hydrogen molecule filled hollow-core photonic crystal fiber (HCPCF) excited with nanosecond optical pulses [4-5]. The fiber single mode guidance allows to act as a "spatial filter" for the Stokes spatial modes,$\phi_{k}(r)$, so only the mode with the highest Raman gain survives the propagation. However, no temporal measurements was reported in this work, and the use of long pulses raises the question on the amplified Stokes temporal modal content, i.e. the number of $\psi_{n}(t)$ that has been amplified from the noise. A means to ensure the Raman amplification of a single Stokes STM from the quantum noise is to use a single mode HCPCF with a short pulse laser, whose combination will act as a temporal-spatial filter, thus favoring one single STM to be amplified.

Here, we report on the observation of a train of sub-20 fs pulses spaced by $57 \mathrm{fs}$ by sending a portion of a spectral comb into an auto-correlator and a frequency-resolved optical gating (FROG). The spectral comb is generated by exciting hydrogen filled single mode Inhibited-Coupling HCPCF (IC-HCPCF) with a single 10 picosecond laser.

\section{Results}

Fig. 1 shows the typical generated Raman spectrum along with the selected portion for temporal detectionhighlighted with dashed yellow rectangle (Fig.1a), and the calculated energy extinction ratio between the second highest gain temporal mode, $\psi_{2}(t)$ over the highest gain mode, $\psi_{1}(t)$ in the laser pulse duration-average power space (Fig.1b). The Raman spectrum is generated using an experimental setup composed of a commercial laser delivering pulses at $1030 \mathrm{~nm}$ with a repetition frequency of $250 \mathrm{kHz}$ up to $36.5 \mathrm{~W}$ average power. The pulse duration is tunable between $250 \mathrm{fs}$ and $10 \mathrm{ps}$. The beam power is controlled by a polarized beam splitter and a half-wave plate and the polarization by a quarter-wave plate. The latter is used to control the excitation of the vibrational or rotational stokes lines of the $\mathrm{H}_{2}$ molecule. The beam is coupled into a $\mathrm{H}_{2}$-filled HCPCF. The latter is an IC-HCPCF with Kagome cladding structure and negative-curvature core contour with a core diameter of 60 $\mu \mathrm{m}$. The fiber length was set to 3 meters and the gas pressure to $20 \mathrm{bar}$. At the fiber output, the beam is analyzed by a detection system composed of a FROG and auto-correlator to measure which allows to measure the spectrum and the pulse train duration over a large range and with high resolution. Two optical spectrum analyzers are used to record the spectrum of the Raman comb over $400 \mathrm{~nm}$ to $2400 \mathrm{~nm}$. The comb shown in Fig. 1a covers over 3 octaves and was obtained with input power of $8 \mathrm{~W}(32 \mu \mathrm{J})$ and a pulse duration of 10 ps. The energy extinction ratio between the two dominant STMs is numerically calculated using the formalism of [3]. The black dashed rectangle shows the duration-power dynamic achievable in our experimental conditions. We note that for a pulse of $10 \mathrm{ps}$ and power of less than $10 \mathrm{~W}$, the energy ratio between the second dominant temporal mode and the first one is less $5 \times 10^{-3}$, corresponding to an extinction ratio of $\sim 50 \mathrm{~dB}$. 
(a)

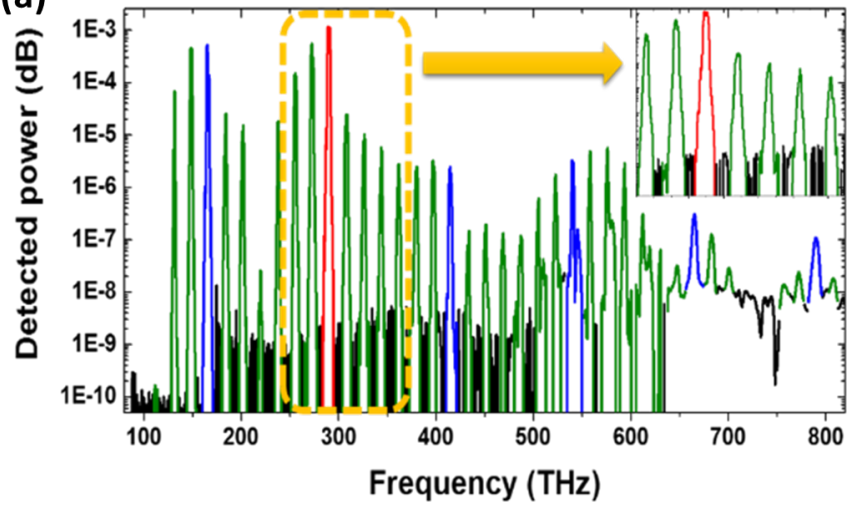

(b)

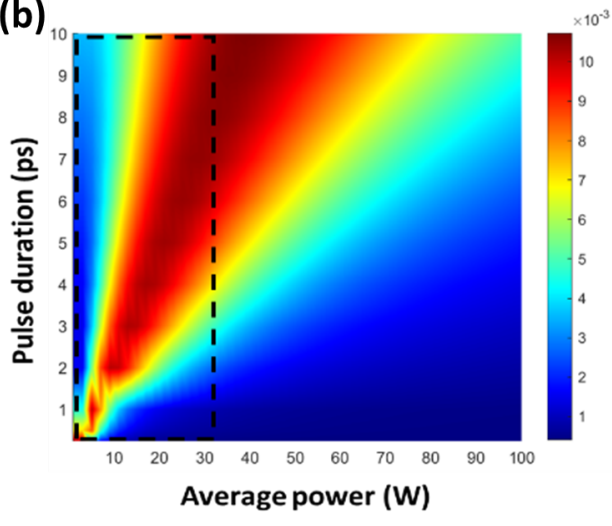

Fig. 1. (a) Typical generated Raman spectrum, obtained with $8 \mathrm{~W}$ average input power and with 10 picosecond pump pulse duration. Inset shows the processed portion for pulse synthesis. (b) Energy ratio between the two dominant Stokes STM versus average power and pulse duration. The area accessible by our laser is shown in dashed black line.
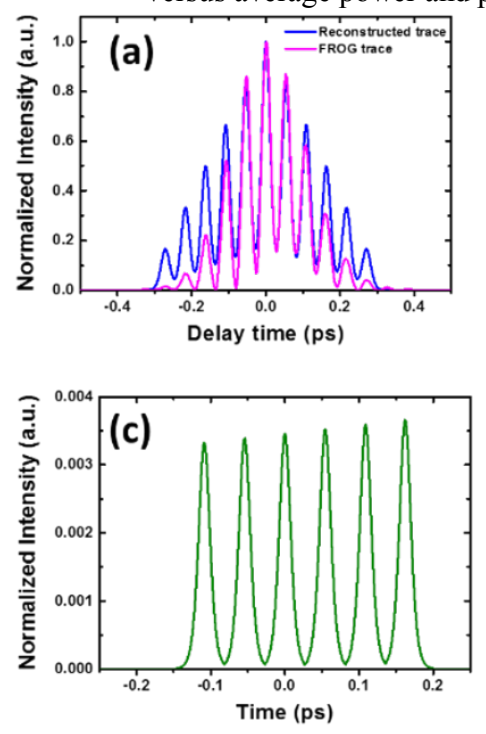
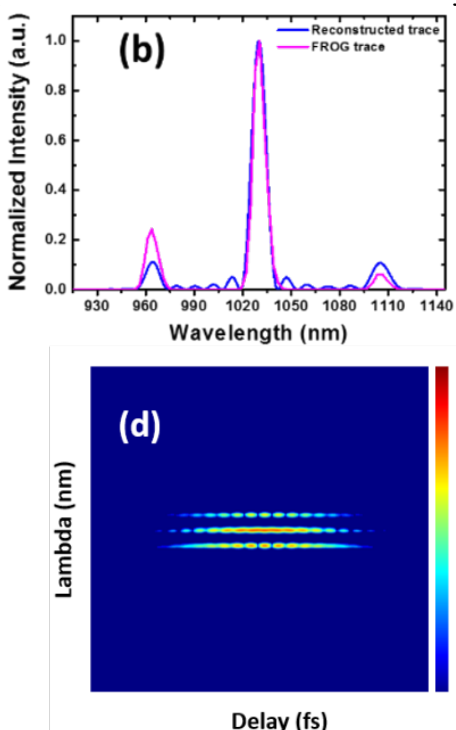

(e)

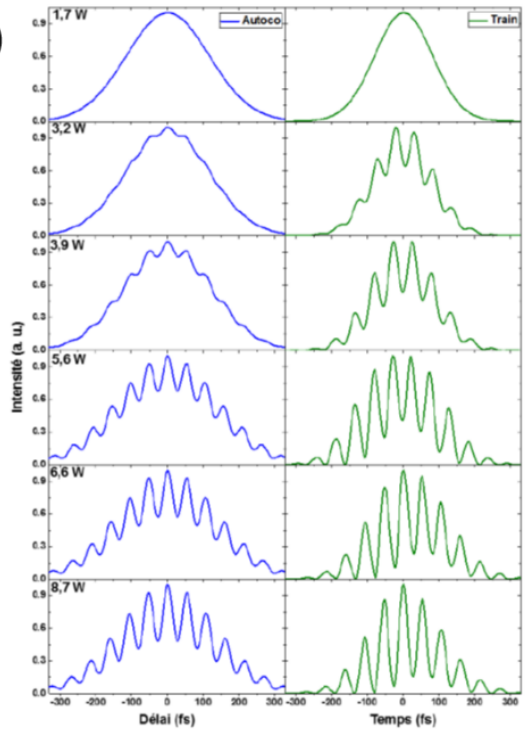

Fig. 2. (a) Example of detected autocorrelation trace (magenta) and the reconstructed one (blue), showing both $57 \mathrm{fs}$ period pulse train. (b) Corresponding detected (magenta) and reconstructed (blue) spectrum. (c) Resulting femtosecond pulse train.

(d) FROG retrieved spectrogram. (e) Pulse train evolution and retrieved autocorrelation trace with power for circular polarization.

Fig. 2 shows an example of FROG measurements with 10 ps pulses and $8 \mathrm{~W}$ of average power. Here both experimental (magenta) and numerically reconstructed (blue) results are plotted. The agreement is found to be good between reconstructed and the autocorrelation traces (Fig. 2(c)). Fig. 2(d) shows the FROG traces of the recorded spectrum. Fig. 2(e) shows the autocorrelation traces and the retrieved pulse train for different input powers at $10 \mathrm{ps}$ and for pump circular polarization. For low power, the traces are that of the pump. As the power is increase the autocorrelation trace exhibit equally spaced peak with higher contrast as the input power is increased. The peak spacing matches the period of the $17.6 \mathrm{THz}$ rotational Raman resonance. The duration of the pulses are found to be less than 20 fs. These results are consistent with the increase of the extinction ratio between the dominant STM with pump power for the range of 1-8W.)

In conclusion, we demonstrated ultrafast pulse train generation with a simple and relatively compact experimental setup. The pulses have a 20 fs duration and a 57 fs period.

\section{References}

[1] S. E. Harris et al., Physical Review Letters, 81(14), p. 2894-2897(1998).

[2]H.S. Chan, et al.,Science, 331(6021), 1165-1168 (2011). (1990).

[3] M.G. Raymer and I.A.Walmsley, Progress in Optics. 28

[4] F Couny, et al., Science 318 (5853), 1118-1121(2007)

[5] YY Wang, et al.,Physical review letters 105 (12), 123603 